\title{
Modeling the non-thermal emission of the gamma Cygni Supernova Remnant up to the highest energies
}

\author{
Henrike Fleischhack* \\ Michigan Technological University \\ E-mail: hfleisch@mtu.edu \\ for the HAWC collaboration ${ }^{\dagger}$
}

The gamma Cygni supernova remnant (SNR) is a middle-aged, Sedov-phase SNR in the Cygnus region. It is a known source of non-thermal emission at radio, X-ray, and gamma-ray energies. Very-high energy (VHE, $>100 \mathrm{GeV}$ ) gamma-ray emission from gamma Cygni was first detected by the VERITAS observatory and it has since been observed by other experiments. Observations so far indicate that there must be a population of non-thermal particles present in the remnant which produces the observed emission. However, it is not clear what kind of particles (protons/ions or electrons) are accelerated in the remnant, how efficient the acceleration is, and up to which energy particles can be accelerated. Accurate measurements of the VHE gamma-ray spectrum are crucial to investigate particle acceleration above $\mathrm{TeV}$ energies.

This presentation will focus on multi-wavelength observations of the gamma Cygni SNR and their interpretation. We will present improved measurements of the VHE gamma-ray emission spectrum of gamma Cygni by the High-Altitude Water Cherenkov (HAWC) gamma-ray observatory, and use these results as well as measurements from other instruments to model the underlying particle populations producing this emission. HAWC's excellent sensitivity at TeV energies and above enables us to extend spectral measurements to higher energies and better constrain the maximum acceleration energy.

36th International Cosmic Ray Conference -ICRC2019-

July 24th - August 1st, 2019

Madison, WI, U.S.A.

\footnotetext{
* Speaker.

${ }^{\dagger}$ Full author list and acknowledgements: PoS(ICRC2019)1177 and http://www. hawc-observatory . org/collaboration/icrc2019.php
} 


\section{Introduction}

The $\gamma$ Cygni SNR $($ G78.2+2.1) is a middle-aged SNR located in the Cygnus region of our Galaxy, named for its location near the star $\gamma$ Cygni. The star is not related to the remnant; in the following text, " $\gamma$ Cygni" will be used to refer to the SNR. Its age has been estimated to be 5 kyr to $10 \mathrm{kyr}[1,2]$, and its distance from Earth to $1.7 \mathrm{kpc}$ to $2.6 \mathrm{kpc}$ [2].

Gamma-ray emission from the $\gamma$ Cygni SNR has been detected both in the $\mathrm{GeV}$ and the $\mathrm{TeV}$ range. The $\mathrm{GeV}$ emission has two components, an extended 'disk' coincicent with the radio shell as well as a hotspot in the northwestern quadrant of the remnant (see e.g. [3, 4, 5, 6]). In the VHE regime, gamma-ray emission was first reported by VERITAS, which detected emission from the north-western hotpot, VER J2019+407 [7]. The MAGIC collaboration has reported significant emission from both the hotspot and the disk component [8].

The Cygnus region, in which $\gamma$ Cygni is located, contains several gamma-ray sources. In particular, $\gamma$ Cygni overlaps with the Cygnus cocoon, an extended source of $\mathrm{GeV}$ to $\mathrm{TeV}$ gamma rays $[9,10]$. In the analysis of HAWC data, care must be taken to dis-entangle the two sources from each other, as not to over-estimate the emission from $\gamma$ Cygni. The multi-source analysis of the $\mathrm{TeV}$ emission from the region is described in [9]

In this study, we will extract the $\mathrm{GeV}$ to TeV energy spectrum of the $\gamma$ Cygni SNR and model it as a pion decay spectrum.

\section{Instruments and Data Analysis}

Data from two instruments was used in this study: The HAWC (High Altitude Water Cherenkov) ground-based gamma-ray Observatory and the Fermi-LAT (Large Area Telescope aboard NASA's Fermi satellite).

HAWC is sensitive to gamma-ray air showers with a primary energy between a few hundred $\mathrm{GeV}$ to hundreds of $\mathrm{TeV}$. Its angular resolution depends mainly on the fraction of the detector hit by a given event, and ranges from $0.10^{\circ}$ to $1^{\circ}$. As an air shower detector, HAWC's main background is due to cosmic-ray induced showers which mimic gamma-ray events. HAWC's data are proprietary; details on data reconstruction and basic analysis can be found in $[12,13]$. The dataset used for this analysis comprises 1038 days of HAWC data, reconstructed with the ground parameter energy estimator and binned according to the two-dimensional scheme described in [14].

HAWC data are analyzed using a binned likelihood prescription as described in [15]. For this study, the threeML framework [16] with the HAL plugin was used for HAWC data analysis as well as joint fits to HAWC and Fermi-LAT data.

The LAT detects gamma rays from $20 \mathrm{MeV}$ to several $\mathrm{TeV}[17,18,19]$. Its angular resolution ranges from several degrees below $100 \mathrm{MeV}$ to about $0.10^{\circ}$ above $30 \mathrm{GeV}^{1}$. The LAT has very good hadron rejection capabilities; for most analyses, the main backgrounds are gamma-ray photons from nearby sources or diffuse emission from the Galactic plane.

For this study, we used 9.8 years of Fermi-LAT data (up to May 15, 2018), reconstructed according to pass 8 revision 2. SOURCE class events in between $1 \mathrm{GeV}$ and $870 \mathrm{GeV}$, originating from the Cygnus region, were analyzed using the fermipy[20] analysis framework.

\footnotetext{
${ }^{1}$ https://www.slac.stanford.edu/exp/glast/groups/canda/lat_Performance.htm
} 

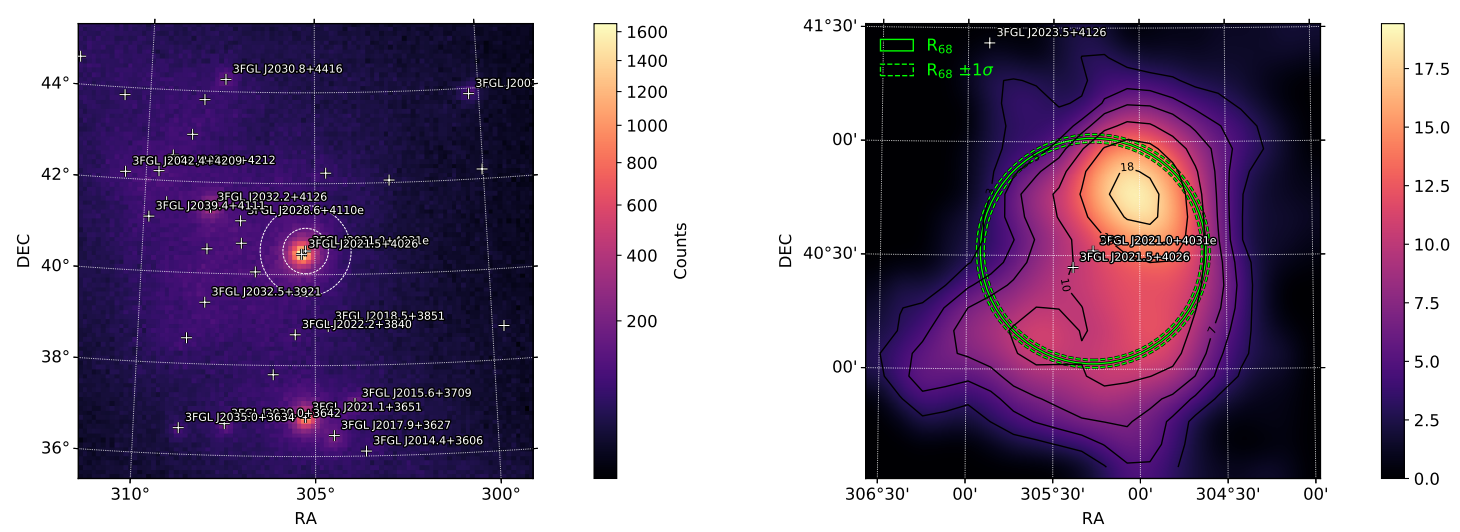

Figure 1: Fermi-LAT gamma-ray maps. Left: Gamma-ray counts above $1 \mathrm{GeV}$, smoothed. Right: TS map of $\gamma$ Cygni after subtracting all other sources in the region (point source assumption, spectral index -2).
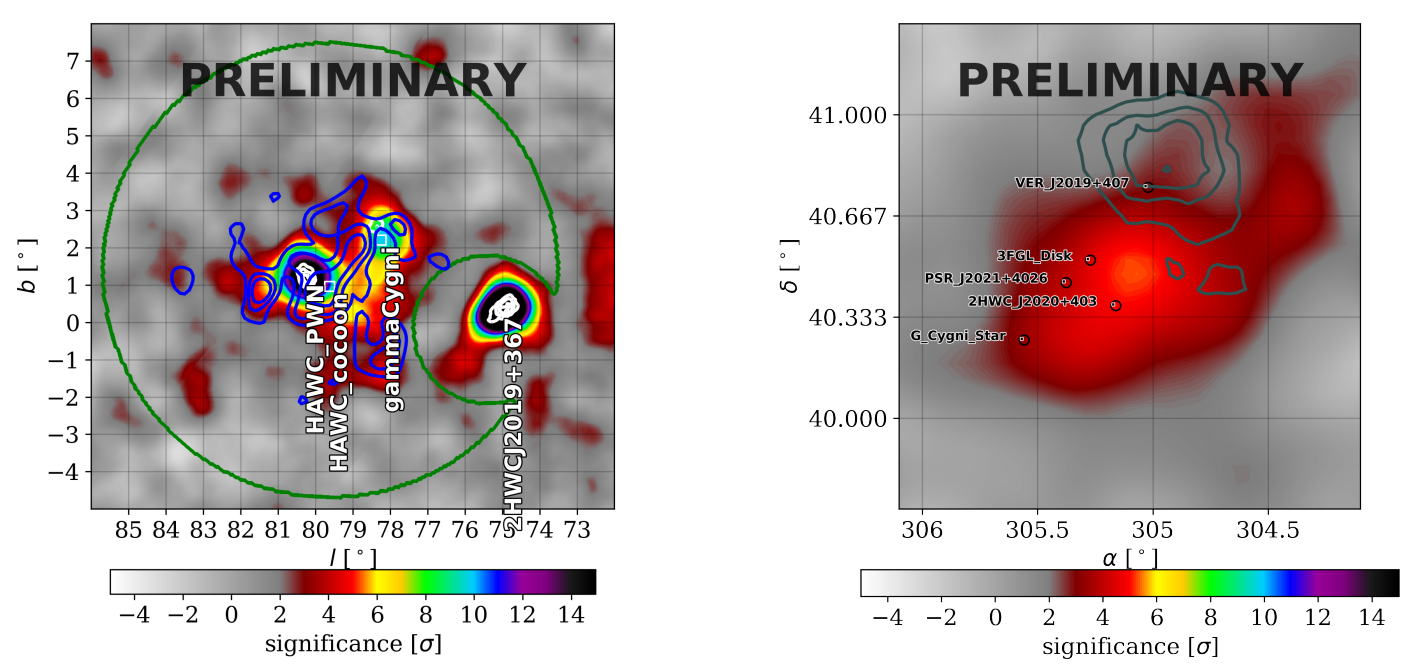

Figure 2: HAWC gamma-ray maps. Left: significance map of the Cygnus cocoon region, with the ROI marked in green. Blue contours: Cygnus cocoon [10], White contours; VERITAS ([11], data provided by R. Bird). Right: Significance map of $\gamma$ Cygni after subtracting all other sources in the model (point source assumption, spectral index -2.7). Grey contours; VERITAS ([11]. 


\section{Modeling of the Region}

For the analysis of the Fermi-LAT data set, data within $10^{\circ}$ of the nominal position of the $\gamma$ Cygni SNR were selected. For the model, all 3FGL [4] sources within $15^{\circ}$ (accounting for photon leakage from sources outside the region of interest [ROI]) of the ROI center were considered, as well as the usual diffuse emission templates. All sources within $5^{\circ}$ of the ROI center and/or with at $T S>10$ in the initial optimize step were freed for the fit. Source positions and morphologies (for extended sources) were fixed to their 3FGL values. In particular, $\gamma$ Cygni (3FGL $\mathrm{J} 2021.0+4031 \mathrm{e}$ ) was modeled as a disk with centroid at RA $305.27^{\circ}$, Dec $40.52^{\circ}$ and radius $0.63^{\circ}$.

The HAWC data mirrors what is described in [9]. The region of interest was chosen to be $6^{\circ}$ around $\mathrm{RA}=307.17^{\circ} \mathrm{Dec}=41.17^{\circ}$, with a $2^{\circ}$ cutout to remove contributions from the bright source 2 HWC J2019+367. The HAWC emission is well described by three components. The extended $\mathrm{TeV}$ counterpart of the cocoon and the counterpart of VER J2031+415 are modeled as described in [9], with their morphologies fixed and spectral parameters free. $\gamma$ Cygni was modeled with a disklike morphology as in the 3FGL, with a power law energy spectrum. For this study, the morphology was fixed.

As in [6], we also considered the presence of a second 'hotspot' component, a possible counterpart of VER J2019+407. For the Fermi-LAT analysis, this was modeled with a gaussian morphology, centered on RA $305.02^{\circ}$, Dec $40.76^{\circ}$, with a $68 \%$ containment radius of $0.23^{\circ}$, and a power law energy spectrum. For the HAWC analysis, this source was modeled as a point source as its extend is small compared to HAWC's angular resolution, again with a power-law energy spectrum.

\section{Gamma-Ray Emission from the $\gamma$ Cygni SNR}

\subsection{Morphology}

In Fermi-LAT data, both the 'disk' and the 'hotspot' component of the $\gamma$ Cygni SNR are significantly detected (TS of 142 for the hotspot and 581 for the disk). Their spectral indices are consistent with each other within uncertainties (see Table 1). HAWC significantly detects the 'disk' component, but there is no preference for including the hotspot. Significance maps derived from both datasets are show in Fig. 1 and Fig. 2.

\subsection{Energy Spectra}

In both the Fermi-LAT and the HAWC data, $\gamma$ Cygni is well fit by power-law energy spectra, with no significant preference for curvature (see Table 1, Fig. 3). Spectral points were extracted for the Fermi-LAT data range using fermipy's sed function. $\gamma$ Cygni is detected with a TS of 39.3 in the HAWC dataset; this detection was not significant enough to extract spectral points.

The two spectra are plotted in Fig. 3. They line up reasonably well with each other, but the $\mathrm{TeV}$ spectrum measured by HAWC is significanly softer than the GeV spectrum, indicating the presence of a spectral break or cutoff. 


\section{Hadronic Modeling}

Assuming that the $\mathrm{GeV}-\mathrm{TeV}$ emission from $\gamma$ Cygni is dominated by hadronic processes (relativistic protons and nuclei produce pions in interactions with the ISM, some of which decay into gamma rays), we attempted to extract the parameters of the underlying proton spectrum. The naima package [21] was used to predict the gamma-ray emission from a given proton spectrum. The threeML framework was used to fit the underlying model parameters to the Fermi-LAT and HAWC data simultaneously. In this case, the HAWC data was read in directly via the hawc_hal plugin, while the spectral points corresponding to the Fermi-LAT measurement of $\gamma$ Cygni were read in via the XYlike plugin. The other two HAWC sources in the region were modeled as described above.

Pion decay emission from a simple power-law proton spectrum would produce gamma-ray emission following a pure power law above about $1 \mathrm{GeV}$, which is not compatible with the observed break in the gamma-ray spectrum. However, a powerlaw spectrum with an exponential cutoff, $\frac{d N}{d E} \propto$ $\left(\frac{E}{E_{0}}\right)^{-\gamma} \cdot \exp \left(-\frac{E}{E_{C}}\right)$, reproduces the $\mathrm{GeV}$ to $\mathrm{TeV}$ data well. The best-fit proton model parameters is described by the following:

- Total energy $W_{p}$ in relativistic protons (above $1 \mathrm{GeV}$ ):

$W_{p}=(1.4 \pm 0.1) \cdot 10^{50} \mathrm{erg} \cdot\left(\frac{2 \mathrm{pc}}{d}\right)^{2} \cdot \frac{1 \mathrm{~cm}^{-3}}{n}$, where $d$ is the distance to the SNR and $n$ is the number density of hydrogen nuclei in the emission region.

- Proton spectral index $\gamma=2.137 \pm 0.033$

- Proton cutoff energy: $E_{C}=(20 \pm 5) \mathrm{TeV}$.

The uncertainties given here are statistical only. Not that there is no cross-calibration between Fermi-LAT and HAWC yet; systematic differences in the flux or energy scales between the two instruments are under investigation and could lead to large uncertainties on the cutoff energy. The resulting gamma-ray energy spectrum can be seen in Fig. 3.

These results are similar to what had been found in [6], which focussed on the hotspot component instead.

\section{Conclusions and Discussion}

HAWC detects VHE gamma-ray emission from the $\gamma$ Cygni SNR, in spatial coincidence with the 'disk' emission seen by Fermi-LAT. The VHE energy spectrum is significantly harder than what is seen at $\mathrm{GeV}$ energies. The resulting $\mathrm{GeV}$ to $\mathrm{TeV}$ spectrum is well-fit by a pion decay model, assuming the underlying proton spectrum is well described by a power-law with an exponential cutoff.

In the future, we plan to repeat the Fermi-LAT analysis of the region, using the new 4FGL catalog [22] and updated diffuse emission models as a baseline. In particular, we plan to look for a pion bump, a characteristic cutoff below $100 \mathrm{MeV}$ indicative of emission from pion decays.

Leptonic emission models are also under consideration. 
Table 1: Fit parameters of the energy spectra of the $\gamma$ Cygni SNR, fit to Fermi-LAT and HAWC data separately. Statistical uncertainties only.

\begin{tabular}{llcccc}
\hline Instrument & Component & $\begin{array}{c}E_{0} \\
{[\mathrm{TeV}]}\end{array}$ & $\begin{array}{c}K \\
{\left[\mathrm{~cm}^{-2} \mathrm{~s}^{-1} \mathrm{TeV}^{-1}\right]}\end{array}$ & $\gamma$ & TS \\
\hline Fermi-LAT & Disk & 0.001 & $(1.2 \pm 0.1) \cdot 10^{-5}$ & $2.06 \pm 0.03$ & 580 \\
& Hotspot & 0.001 & $(2.8 \pm 0.6) \cdot 10^{-6}$ & $2.11 \pm 0.09$ & 142 \\
\hline Fermi-LAT & Disk & 0.001 & $(1.50 \pm 0.06) \cdot 10^{-5}$ & $2.06 \pm 0.02$ & 767 \\
\hline HAWC & Disk & 2.6 & $(3 \pm 0.5) \cdot 10^{-13}$ & $3.02 \pm 0.14$ & 39.3 \\
\hline
\end{tabular}

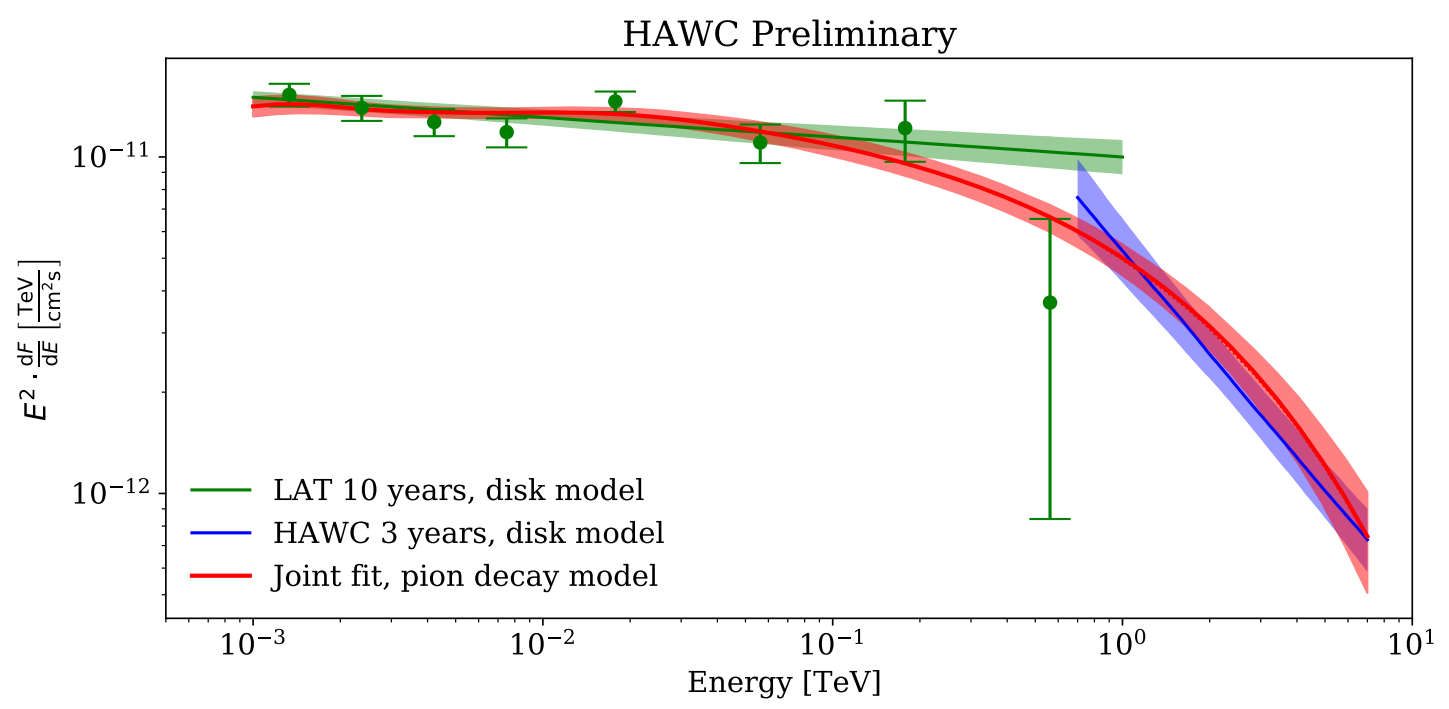

Figure 3: Gamma-ray energy spectra of $\gamma$ Cygni. Statistical uncertainties only. 


\section{References}

[1] T. A. Lozinskaya, V. V. Pravdikova \& A. V. Finoguenov. "The Supernova Remnant G78.2+2.1: New Optical and X-ray Observations". In: Astronomy Letters 26 (Feb. 2000), pp. 77-87.

[2] D. A. Leahy, K. Green \& S. Ranasinghe. "X-ray and radio observations of the Îs Cygni supernova remnant G78.2+2.1". In: Monthly Notices of the Royal Astronomical Society 436.2 (Oct. 2013), pp. 968-977.

[3] F. Acero et al. "THE FIRST FERMILAT SUPERNOVA REMNANT CATALOG". In: The Astrophysical Journal Supplement Series 224.1 (2016), p. 8.

[4] F. Acero et al. "FERMI LARGE AREA TELESCOPE THIRD SOURCE CATALOG". In: The Astrophysical Journal Supplement Series 218.2 (2015), p. 23.

[5] M. Ackermann et al. "Search for Extended Sources in the Galactic Plane Using Six Years of Fermi-Large Area Telescope Pass 8 Data above 10 GeV”. In: Astrophys. J. 843.2 (2017), p. 139.

[6] N. Fraija \& M. Araya. "The GeV counterpart of VER J2019+407 in the northern shell of the supernova remnant G78.2+2.1 ( $\gamma$ Cygni)”. In: Astrophys. J. 826.1 (2016), p. 31.

[7] E. Aliu et al. "Discovery of TeV Gamma-ray Emission Toward Supernova Remnant SNR G78.2+2.1”. In: Astrophys. J. 770 (2013), p. 93.

[8] M. Strzys. "Gamma-Cygni: the GeV to TeV morphology of an unique Sedov-phase SNR with MAGIC andF ermi-LAT". In: PoS ICRC2017 (2017).

[9] B. Hona et al. "Testing the Limits of Particle Acceleration in Cygnus OB2 with HAWC". PoS(ICRC2019)699. In: These proceedings. 2019.

[10] M. Ackermann et al. "A Cocoon of Freshly Accelerated Cosmic Rays Detected by Fermi in the Cygnus Superbubble”. In: Science 334.6059 (2011), pp. 1103-1107.

[11] A. U. Abeysekara et al. "A Very High Energy $\gamma$-Ray Survey toward the Cygnus Region of the Galaxy". In: The Astrophysical Journal 861.2 (2018), p. 134.

[12] A. U. Abeysekara et al. "The 2HWC HAWC Observatory Gamma-Ray Catalog”. In: The Astrophysical Journal 843.1 (2017), p. 40.

[13] A. U. Abeysekara et al. "Observation of the Crab Nebula with the HAWC Gamma-Ray Observatory". In: Astrophys. J. 843.1 (2017), p. 39.

[14] A. U. Abeysekara et al. "Measurement of the Crab Nebula at the Highest Energies with HAWC". In: Accepted for publication by ApJ (2019).

[15] P. W. Younk et al. "A high-level analysis framework for HAWC". In: Proceedings of the 34th International Cosmic Ray Conference (PoS). 2015, arXiv:1508.07479.

[16] G. Vianello et al. "The Multi-Mission Maximum Likelihood framework (3ML)". In: Proceedings of the 34th International Cosmic Ray Conference (PoS). 2015.

[17] W. B. Atwood et al. "The Large Area Telescope on the Fermi Gamma-Ray Space Telescope Mission”. In: ApJ 697.2 (2009), pp. 1071-1102. 
[18] P. Bruel et al. "Fermi-LAT improved Pass $\sim 8$ event selection". In: arXiv e-prints, arXiv:1810.11394 (2018), arXiv:1810.11394.

[19] W. Atwood et al. "Pass 8: Toward the Full Realization of the Fermi-LAT Scientific Potential”. In: arXiv e-prints, arXiv:1303.3514 (2013), arXiv:1303.3514.

[20] M. Wood et al. "Fermipy: An open-source Python package for analysis of Fermi-LAT Data". In: International Cosmic Ray Conference 35, 824 (Jan. 2017), p. 824.

[21] V. Zabalza. "naima: a Python package for inference of relativistic particle energy distributions from observed nonthermal spectra". In: Proc. of International Cosmic Ray Conference 2015 (2015), p. 922.

[22] The Fermi-LAT collaboration. "Fermi Large Area Telescope Fourth Source Catalog". In: arXiv e-prints, arXiv:1902.10045 (2019), arXiv:1902.10045. 